Revista Brasileira de Agricultura Irrigada v.8, nº 3, p. 247 - 255, 2014

ISSN 1982-7679 (On-line)

Fortaleza, CE, INOVAGRI - http://www.inovagri.org.br

DOI: $10.7127 /$ rbai.v8n300197

Protocolo 197/13 - 04/11/2013 Aprovado em 25/04/2014

\title{
SAZONALIDADE NA QUALIDADE DA ÁGUA DE IRRIGAÇÃO EM AÇUDES DA BACIA DO RIO ACARAÚ, CEARÁ
}

\author{
Waleska Martins Eloi ${ }^{1}$, Mariana Alexandre de Lima Sales ${ }^{2}$, Jeniffer Vasconcelos de \\ Lira $^{3}$, Maria Leila Mesquita Sales ${ }^{4}$, Navilta Veras do Nascimento ${ }^{5}$, João Victor Ribeiro da \\ Silva de Souza ${ }^{6}$
}

\begin{abstract}
RESUMO
O conhecimento da concentração de sais e consequentemente a qualidade da água para irrigação é de extrema importância no manejo da irrigação uma vez que pode limitar o seu uso, alterando severamente as características químicas e físicas do solo. O presente trabalho foi realizado na Bacia do Rio Acaraú, localizada na zona Noroeste do Estado do Ceará. Foram realizadas cinco amostragens ao longo de um ano de seis açudes da região, no período de Agosto de 2009 a Julho de 2010 e em cada ponto a coleta foi realizada utilizando-se quatro repetições tendo ao final 120 amostras ao longo de todo o período e todos os pontos foram georrefenciados. Avaliaram-se os parâmetros: condutividade elétrica da água (CEa), potencial hidrogeniônico $(\mathrm{pH})$, cátions $\left(\mathrm{Ca}^{2+}, \mathrm{Mg}^{2+}, \mathrm{Na}^{+} \mathrm{e} \mathrm{K}^{+}\right)$e ânions. Em geral águas que contêm concentrações elevadas de íons de bicarbonato, tem uma grande tendência para a precipitação de $\mathrm{Ca}$ e $\mathrm{Mg}$, as águas dos açudes estudados entre o período de setembro de 2009 e abril de 2010 encontram-se, com os valores normais para irrigação, exceto para os parâmetros cálcio, magnésio, cloreto e carbonatos. Este estudo mostra a necessidade de monitoramento desta bacia para tomadas de decisões e elaboração de ações e políticas publicas agrícolas para o melhor desempenho da agricultura local, uma vez que as condições climáticas predominantes na região analisada favorecem a um maior risco de salinidade e sodicidade ao longo do ano no período de temperaturas mais elevadas.
\end{abstract}

Palavras-chave: salinidade, irrigação, variabilidade espacial

\footnotetext{
${ }^{1}$ Engenheira Agrônoma, Prof ${ }^{a}$ Dra. Departamento Construção Civil, Instituto Federal do Ceará, Avenida Treze de Maio, 2081 - Benfica, Fortaleza - CE, 60040-531, Fortaleza-CE. e-mail: waleskaeloi@msn.com

${ }^{2}$ Mestranda em Agronomia: Irrigação e Drenagem, FCA/UNESP, Dept. de Eng. Rural, Fazenda Experimental

Lageado - Rua José Barbosa de Barros, 1780 - CEP 18610-307 - Botucatu-SP. e-mail: mal_sales@ @otmail.com

${ }^{3}$ Tecnologa em Irrigação e Drenagam. Rua Cel. Estanislau Frota, № 118, Centro, CEP 62010-560. Sobral-CE.email: jeninhalegiao@ hotmail.com

${ }^{4}$ Especialista em Vigilância Sanitária e Meio Ambiente. Av. Dr. Guarany, 317. Bairro Derby Clube. CEP 62040-030. Sobral-CE. e-mail: leilaifce@yahoo.com.br

${ }^{5}$ Doutoranda em Engenharia Agrícola, UFCG-Campus I, Campina Grande, PB. Rua Eva, no 294, Apt 102,bloco B, Bairro da expectativa. CEP 62040-040. Sobral-CE. e-mail: naviltaveras@ gmail.com

${ }^{6}$ Mestrando em Agronomia: Irrigação e Drenagem, FCA/UNESP, Dept. de Eng. Rural, Fazenda Experimental

Lageado - Rua José Barbosa de Barros, 1780 - CEP 18610-307 - Botucatu-SP. e-mail: mim_joao@hotmail.com
} 


\title{
SEASONALITY IN THE QUALITY OF IRRIGATION WATER IN DAMS ACARAÚ RIVER BASIN, CEARÁ
}

\begin{abstract}
Knowledge of salt concentration and consequently the quality of irrigation water is of utmost importance in irrigation management since it may limit its use, severely altering the chemical and physical characteristics of the soil . This study was conducted at Bacia do Rio Acaraú, located in the northwest of the state of Ceará. Five samples were collected over a year of six ponds in the region, in the period August 2009 to July 2010 and on each collection was performed using four replications with the end 120 samples throughout the period and all points were geo-referenced . Evaluated parameters were : electrical conductivity of water $(\mathrm{ECw})$, hydrogen potential $(\mathrm{pH})$, cations $\left(\mathrm{Ca}^{2+}, \mathrm{Mg}^{2+}, \mathrm{Na}^{+}\right.$and $\left.\mathrm{K}^{+}\right)$and anions . Generally waters containing high concentrations of bicarbonate ions, has a great tendency for precipitation of $\mathrm{Ca}$ and $\mathrm{Mg}$, the waters of the dams studied in the period between September 2009 and April 2010 are , with normal irrigation except for the parameters calcium, magnesium, chloride and carbonate. This study shows the need for monitoring of the basin for decision making and development of agricultural activities and public policies for the better performance of local agriculture, since the climatic conditions prevailing in the analyzed region favor a higher risk of salinity and sodicity along the year from higher temperatures
\end{abstract}

Key words: salinity, irrigation, spatial variability

\section{INTRODUÇÃO}

A água é vital para a sobrevivência humana e seu consumo é essencial em diversas atividades, sendo de suma importância a analise da sua qualidade para a correta utilização (ELOI \& BARRETO, 2011). De acordo com Cordeiro (2001), os estudos visando determinar a sua qualidade, sob o ponto de vista de sua utilização na agricultura irrigada, englobam recursos utilizados para indicar a conveniência ou limitação de seu emprego para fins de irrigação. Toda água usada na irrigação contém sais dissolvidos, o efeito destes sais sobre as características químicas e físicas de solos irrigados é de grande importância para manutenção da sua capacidade produtiva.

Na prática da irrigação, a longo prazo, a qualidade da água é um dos fatores mais importantes, pois pequenas quantidade de soluto podem, em projetos de irrigação mal elaborados, transformar lentamente uma área fértil em um solo salino de baixa produtividade, e se não existir um monitoramento constante da qualidade da água utilizada, pode-se apenas perceber o problema em uma condição bem evoluída, sendo muitas vezes tarde demais, pois a recuperação de solos salinos ou salinizados é difícil, demorada e dispendiosa (REICHARDT, 1990).

Os parâmetros de qualidade da água que devem ser avaliados englobam valores diferenciados, de acordo com a sua utilização, assim os aspectos físicos, químicos e biológicos devem ser analisados de acordo com a destinação do recurso (ELOI \& BARRETO, 2011)

A qualidade da água é avaliada dependendo das circunstâncias de uso, para irrigação se avaliam o potencial da água em causar salinização, a redução na capacidade de infiltração da água no solo, a concentração 
elevada de íons específicos $(\mathrm{Cl}, \mathrm{Na}$ e B) que causam toxicidade nas plantas e os efeitos indiretos como aqueles que provocam problemas nutricionais nas plantas (GHEYI et al., 2012).

Vários autores têm proposto esquemas de classificação da água para irrigação. A FAO recomenda a classificação proposta pela Univesity of California Committee of Consultants (1974), divulgada por Ayres e Westcot (1999), cujas diretrizes levam em consideração o estudo do grau de restrição no uso de águas com problemas potenciais para salinização do solo, redução de infiltração de água no solo, toxidade de íons específicos e outros problemas.

A avaliação da água para irrigação fundamenta-se na identificação de suas características químicas e de possíveis problemas relativos ao risco de salinização dos solos, de infiltração e de toxidade de íons, indicando se a água pode ou não ser utilizada para irrigação (SANTOS, 2000).

Segundo Ayers e Westcot (1999) na avaliação da qualidade da água para irrigação, não se deve estudar os parâmetros que determinam sua adequabilidade isoladamente, mas levar em consideração o conjunto de fatores relacionados com suas consequências no solo, na cultura e com o manejo da água e do solo.

A bacia do Acaraú localizada na zona noroeste do Estado do Ceará, região drenada exclusivamente pelo rio Acaraú e seus afluentes, ocupa uma área da ordem de $14.427 \mathrm{~km}^{2}$ que representa $9,22 \%$ da área do Estado. Os rios Groaíras, Jacurutu, dos Macacos e Jaibaras são os principais contribuintes do Acaraú, e compõem a segunda bacia independente do Ceará. Seus 684 açudes conferem uma capacidade de acumulação estimada em 1,6 bilhão de $\mathrm{m}^{3}$, destacando-se dez açudes estratégicos que armazenam 1,37 bilhão de $\mathrm{m}^{3}$ (IPECE, 2002). Um dos principais usos das águas da bacia é o da irrigação com 13 perímetros irrigados, somando uma área de 24.065 ha. Esta área é equivalente a $28,3 \%$ de toda a área irrigada do Estado. O estudo da qualidade da água usada para irrigação nesses açudes é de suma importância, haja vista que as mudanças climáticas ocorridas ao longo do ano ocasionam variações na qualidade da água, o que implicará em manejos diferenciados de uso, em função dos resultados obtidos.

Diante disso, o presente trabalho objetiva verificar o efeito da sazonalidade sobre a qualidade da água usada para irrigação em açudes localizados na Bacia do Rio Acaraú e suas possíveis limitações de uso para irrigação, ao longo do ano, visando um planejamento adequado de manejo desse recurso, com base nos resultados obtidos.

\section{MATERIAL E MÉTODOS}

A pesquisa foi realizada na Bacia do Rio Acaraú. O clima da região onde a bacia se localiza é do tipo Semiárido Quente com precipitações médias anuais entre 600 a $800 \mathrm{~mm}$, entre janeiro e maio as temperaturas são mais elevadas, onde a média fica em torno de $27^{\circ} \mathrm{C}$.

Para a realização do estudo inicialmente foi efetuado o cadastro dos principais açudes (Acaraú Mirim, Araras Norte, Ayres de Sousa, Edson Queiroz, Forquilha, Sobral), com o georreferenciamento dos pontos de coletas (Tabela 01).

Foram realizadas cinco amostragens ao longo de um ano, no período de Agosto de 2009 a Julho de 2010 (período 1 - Setembro; período 2 Dezembro; período 3 - Fevereiro; período 4 Abril; período 5 - julho). Em cada ponto amostrado a coleta foi realizada utilizando-se quatro repetições (R1, R2, R3 e R4 - no mesmo 
dia) finalizando com 120 amostras ao longo de todo o período.

Tabela 1. Latitudes, longitudes e altitudes de cada ponto de coleta

\begin{tabular}{|c|c|c|c|}
\hline Açude & Latitude & Longitude & Altitude \\
\hline Acaraú Mirim & $3^{\circ} 30^{\prime} 29^{\prime \prime} \mathrm{S}$ & $40^{\circ} 16^{\prime} 45^{\prime \prime} \mathrm{W}$ & $49 \mathrm{~m}$ \\
\hline Araras Norte & $4^{\circ} 12^{\prime} 48^{\prime \prime} \mathrm{S}$ & $40^{\circ} 26^{\prime} 09^{\prime \prime} \mathrm{W}$ & $135 \mathrm{~m}$ \\
\hline $\begin{array}{l}\text { Ayres de } \\
\text { Sousa }\end{array}$ & $3^{\circ} 46^{\prime} 54^{\prime \prime} \mathrm{S}$ & $40^{\circ} 29^{\prime} 53^{\prime \prime} \mathrm{W}$ & $90 \mathrm{~m}$ \\
\hline $\begin{array}{l}\text { Edson } \\
\text { Queiroz }\end{array}$ & $4^{\circ} 13^{\prime} 16^{\prime \prime} \mathrm{S}$ & $40^{\circ} 04^{\prime} 08^{\prime \prime} \mathrm{W}$ & $185 \mathrm{~m}$ \\
\hline Forquilha & $3^{\circ} 47^{\prime} 55^{\prime \prime} \mathrm{S}$ & $40^{\circ} 15^{\prime} 21^{\prime \prime} \mathrm{W}$ & $73 \mathrm{~m}$ \\
\hline Sobral & $3^{\circ} 39^{\prime} 22^{\prime \prime} \mathrm{S}$ & $40^{\circ} 21^{\prime} 54^{\prime \prime} \mathrm{w}$ & $74 \mathrm{~m}$ \\
\hline
\end{tabular}

Em cada ponto georreferenciado a coleta da água foi realizada a profundidade de um metro, sendo a água coletada e acondicionada em garrafas plásticas (Figura 01) de um litro e estas encaminhadas para o Laboratório de Água e Solo do IFCE Campus de Sobral.

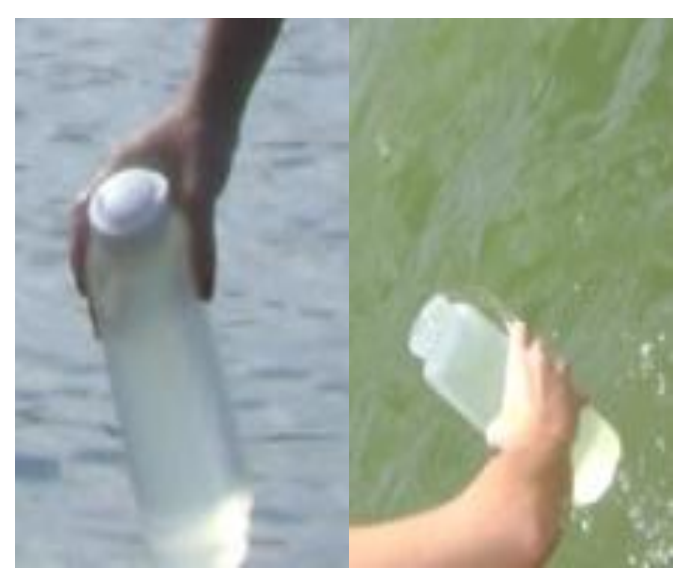

Figura 1. Garrafas utilizadas nas coletas da água.

As análises foram realizadas avaliando-se os seguintes parâmetros: condutividade elétrica da água (CEa), potencial hidrogeniônico $(\mathrm{pH})$, cátions $\left(\mathrm{Ca}^{2+}, \mathrm{Mg}^{2+}, \mathrm{Na}^{+}\right.$e $\left.\mathrm{K}^{+}\right)$e ânions $\left(\mathrm{Cl}^{-}\right.$, $\mathrm{HCO}_{3}{ }^{-}$e $\mathrm{SO}_{4}{ }^{-}$) de acordo com a metodologia descrita por Richards (1954) a qual é a metodologia de classificação de água para irrigação mais utilizada em todo o mundo, onde foi utilizados os equipamentos descrito abaixo (Tabela 02).

Tabela 2. Variáveis e métodos utilizados para a caracterização da água

\begin{tabular}{|c|c|}
\hline Parâmetro & Método \\
\hline Condutividade elétrica & Condutimetria \\
\hline $\begin{array}{c}\text { Potencial } \\
\text { hidrogeniônico }\end{array}$ & Potenciometria \\
\hline Cálcio e Magnésio & Titulometria com EDTA \\
\hline Sódio e Potássio & $\begin{array}{l}\text { Fotometria de emissão de } \\
\text { chamas }\end{array}$ \\
\hline Cloreto & Titulometria com nitrato de prata \\
\hline $\begin{array}{l}\text { Carbonato e } \\
\text { bicarbonato }\end{array}$ & Titulometria com ácido sulfúrico \\
\hline Sulfato & Espectrofotômetria \\
\hline Sólidos dissolvidos & Calcinação \\
\hline
\end{tabular}

De posse dos dados verificou-se as possíveis limitações de uso da água para irrigação, baseado na $\mathrm{CE}$, como indicadora do perigo de salinização, e na relação de adsorção de sódio (RAS), como indicadora do perigo de sodificação do solo, onde a partir dos valores de $\mathrm{Na}^{+}, \mathrm{Ca}^{2+}$ e $\mathrm{Mg}^{2+}$, calculou-se a (RAS). Após as analises, os resultados foram avaliados de acordo com a metodologia descrita por Richards (1954) e Ayres e Westcot (1999).

Com base na concentração desses íons citados acima, estimaram-se os valores de carbonato de sódio residual (CSR) proposto por Eaton, (1949) é a relação de adsorção de sódio 
(RAS) apresentada por Yaron, (1973), modelos apresentados respectivamente nas eq 1 e 2 .

$$
\mathrm{CSR}=\left(\mathrm{CO}_{3}+\mathrm{HCO}_{3}\right)-(\mathrm{Ca}+\mathrm{Mg})
$$

onde as concentrações de $\mathrm{CO}_{3}, \mathrm{HCO}_{3}, \mathrm{Ca}, \mathrm{Mg}$ na água expressas em $\mathrm{mmol}_{\mathrm{c}} \mathrm{L}^{-1}$.

$$
R A S=\frac{N a}{\sqrt{\frac{C a+M g}{2}}}
$$

em que as concentrações de $\mathrm{Na}, \mathrm{Ca}$ e $\mathrm{Mg}$ na água foram expressas em $\mathrm{mmol}_{\mathrm{c}} \mathrm{L}^{-1}$.

\section{RESULTADOS E DISCUSSÃO}

\section{A) Carbonato de Sódio Residual}

Águas que contêm concentrações elevadas de íons de bicarbonato, tem uma grande tendência para a precipitação de $\mathrm{Ca}$ e $\mathrm{Mg}$, ocasionando o entupimento de emissores principalmente na irrigação localizada por gotejamento. Esta propriedade também reduz a concentração de cálcio e magnésio na solução do solo e, consequentemente, aumenta a proporção de sódio no solo.

Pode-se observar na Tabela 03 que não houve problemas com a CSR em nenhum dos açudes e épocas coletadas, pois segundo Wilcox et al. (1954) água com CSR até $1,5 \mathrm{mmol} \mathrm{L}^{-1}$ não oferece nenhum perigo.
Tabela 3. CSR obtidos na análise de água para irrigação

\begin{tabular}{cccccc}
\hline \multirow{2}{*}{$\begin{array}{c}\text { Pontos de } \\
\text { Coleta }\end{array}$} & \multicolumn{5}{c}{ CSR - Carbonato de Sódio Residual $\left(\mathrm{mmol}_{\mathrm{c}} \mathrm{L}^{-}\right.$} \\
\cline { 2 - 6 } & $\begin{array}{c}\text { Período } \\
1\end{array}$ & $\begin{array}{c}\text { Período } \\
2\end{array}$ & $\begin{array}{c}\text { Período } \\
3\end{array}$ & $\begin{array}{c}\text { Período } \\
4\end{array}$ & $\begin{array}{c}\text { Período } \\
5\end{array}$ \\
\hline P1 & 0,46 & 0,7 & 0,23 & 0,33 & 0,30 \\
P2 & 0,39 & 1,3 & 1,10 & 0,81 & 0,89 \\
P3 & 0,73 & 0,81 & 0,47 & 0,53 & 0,86 \\
P4 & 0,24 & 1,22 & 1,22 & 0,87 & 0,99 \\
P5 & 0,95 & 1,35 & 1,07 & 1,09 & 1,12 \\
P6 & 0,73 & 0,99 & 0,73 & 0,89 & 0,82 \\
\hline
\end{tabular}

\section{B) Sólidos Dissolvidos}

De acordo com os resultados obtidos neste trabalho (Tabela 4), pode-se observar que os valores obtidos encontram-se com seus valores baixos. Não oferecendo nenhum risco na sua utilização.

Tabela 4. Concentração de sólidos dissolvidos

\begin{tabular}{|c|c|c|c|c|c|}
\hline \multirow{2}{*}{$\begin{array}{l}\text { Pontos } \\
\text { de } \\
\text { Coleta }\end{array}$} & \multicolumn{5}{|c|}{ Sólidos } \\
\hline & $\begin{array}{c}\text { Período } \\
1\end{array}$ & $\begin{array}{c}\text { Período } \\
2\end{array}$ & $\begin{array}{c}\text { Período } \\
3\end{array}$ & $\begin{array}{c}\text { Período } \\
4\end{array}$ & $\begin{array}{c}\text { Período } \\
5\end{array}$ \\
\hline $\mathrm{P} 1$ & 105 & 67,25 & 107,50 & 93,50 & 197,50 \\
\hline $\mathrm{P} 2$ & 24,25 & 65,25 & 102,50 & 80,00 & 118,75 \\
\hline P3 & 61,00 & 28,50 & 102,75 & 63,75 & 81,00 \\
\hline P4 & 60,67 & 37,00 & 59,50 & 74,25 & 103,00 \\
\hline P5 & 64,75 & 32,25 & 45,25 & 99,50 & 119,00 \\
\hline P6 & 67,25 & 51,75 & 152,50 & 121,50 & 167,75 \\
\hline
\end{tabular}
(mg.L $\left.\mathrm{L}^{-1}\right)$ 
Segundo a resolução do CONAMA (2005), com relação aos Sólidos dissolvidos totais estas coletas de água estariam totalmente de acordo para a produção de hortaliças para o consumo humano, pois não houve valores que ultrapassem $500 \mathrm{mg} \mathrm{L}^{-1}$

Resultado semelhante foi encontrado no trabalho realizado por Moura et al. (2010), onde nenhuma amostra apresentou risco de danos ao sistema de irrigação, sendo que o valor mínimo encontrado de $87,0 \mathrm{mg} \mathrm{L}^{-1}$ no ponto 2 , já o valor máximo observado foi $145 \mathrm{mg} \mathrm{L}^{-1}$ no ponto 1 .

\section{C) RAS e CE}

A condutividade elétrica (CE) é o parâmetro mais utilizado para expressar a salinidade da água e/ou do solo, enquanto a RAS se refere ao risco de sodicidade. Com estes dois parâmetros se pode avaliar os riscos com relação a problemas de infiltração de água no solo.

Analisando os resultados isoladamente dos valores da RAS e da CE (Tabela 05), verifica-se que quanto a CE não apresenta nenhum grau de restrição, pois esta encontrar-se inferior a $0,7 \mathrm{dS}$ $\mathrm{m}^{-1}$; já em relação a RAS observa-se que varia ao longo do ano, apresentando momentos com nenhum, moderado ou severo grau de restrição ao seu uso na irrigação, pois estes valores variaram de 0,98 a 9,6 mmolc $\mathrm{L}^{-1}$

Analisando conjuntamente os valores da RAS e da CE, de acordo com o diagrama para classificação de águas para irrigação, proposto por Richards (1954), a água encontra-se classificada como C1S1, caracterizando-se por apresentar salinidade baixa, e com baixa concentração de sódio, são consideradas de boa qualidade. Os resultados obtidos permitem recomendar a utilização desta água para irrigação na maioria das culturas e solos, com pouca probabilidade de ocasionar salinidade. Alguma lixiviação é necessária, mas isso ocorre nas praticas normais de irrigação, à exceção dos solos com permeabilidade extremamente baixa. Assim, esta água pode ser usada para irrigação em quase todos os tipos de solos, com pequena possibilidade de alcançar níveis indesejáveis de sódio trocável. Exceto a água do ponto 6 (açude Sobral) no período da quarta coleta, que se encontra na Classe $\mathrm{C} 2 \mathrm{~S} 1$, onde está água apresenta salinidade média, esta água pode ser utilizada sempre que houver grau moderado de lixiviação. Plantas com moderada tolerância aos sais podem ser cultivadas, na maioria dos casos, sem práticas especiais de controle da salinidade.

A capacidade de infiltração de um solo cresce com o aumento de sal salinidade e decresce com o aumento da razão de adsorção de sódio (RAS) e, ou, com o decréscimo de sua salinidade. Assim, os dois parâmetros, RAS e salinidade, devem ser analisados conjuntamente para se avaliar corretamente o efeito da água de irrigação na redução da capacidade de infiltração de um solo (Bernardo et al., 2006).

Analisando conjuntamente os parâmetros de CEa e RAS, encontrou-se para CEa valores entre $0,12-0,23 \mathrm{dS} \mathrm{m}^{-1}$ e a RAS entre $0,98 \mathrm{e}$ 9,60, onde de acordo com Ayres e Westcot (1999), esta água apresentou durante todo período avaliado, redução severa na capacidade de infiltração de água no solo respectivamente pelo uso da água na irrigação.

Tabela 5. Relação dos valores de CE $\left(\mathrm{dS} \mathrm{m} \mathrm{m}^{-1}\right)$ e de RAS (mmolc L ${ }^{-1}$ ).

\begin{tabular}{cccccccccccc}
\hline $\begin{array}{c}\text { Pontos } \\
\text { de } \\
\text { Coleta }\end{array}$ & Período 1 & & Período 2 & & Período 3 & Período 4 & Período 5 \\
\hline P1 & RAS & CE & RAS & CE & RAS & CE & RAS & CE & RAS & CE \\
\hline P2 & 0,98 & 0,15 & 5,45 & 0,16 & 4,98 & 0,17 & 3,13 & 0,12 & 2,98 & 0,17 \\
P3 & 2,14 & 0,12 & 4,92 & 0,12 & 3,18 & 0,13 & 2,49 & 0,14 & 4,93 & 0,14 \\
P4 & 3,06 & 0,21 & 6,32 & 0,21 & 4,86 & 0,21 & 3,61 & 0,18 & 3,59 & 0,21 \\
P5 & 3,14 & 0,18 & 5,43 & 0,20 & 5,55 & 0,21 & 3,26 & 0,17 & 3,44 & 0,21 \\
P6 & 4,84 & 0,21 & 9,60 & 0,23 & 5,55 & 0,23 & 5,22 & 0,27 & 6,26 & 0,25 \\
\hline
\end{tabular}


Resultado semelhante em relação a CE foi observada no trabalho de Reis et al. (2011) os quais os resultados apresentaram valores aceitáveis para água de irrigação, com média de $0,02 \mathrm{dS} \mathrm{m}^{-1}$.

\section{D) Potencial Hidrogeniônico (pH)}

Na tabela 6 podemos observar os valores do $\mathrm{pH}$, os quais encontra-se na faixa normal, pois ele variou entre 7,67 e 8,09 (faixa normal entre 6,5 a $8,4)$, de acordo com as diretrizes para interpretação da qualidade de água para irrigação de Ayres \& Westcoot (1999).

Tabela 6. $\mathrm{pH}$

\begin{tabular}{cccccc}
\hline & & \multicolumn{5}{c}{$\mathrm{pH}$} \\
$\begin{array}{c}\text { Pontos } \\
\text { de } \\
\text { Coleta }\end{array}$ & Período 1 & Período 2 & Período 3 & Período 4 & Período 5 \\
\cline { 3 - 6 } & & & & & \\
P1 & 8,09 & 7,85 & 7,80 & 7,85 & 8,07 \\
P2 & 8,07 & 7,78 & 7,86 & 7,80 & 7,94 \\
& & & & & \\
P3 & 8,09 & 7,71 & 7,68 & 7,67 & 7,86 \\
P4 & 8,04 & 7,90 & 8,03 & 7,80 & 7,99 \\
& & & & & \\
P5 & 8,03 & 7,95 & 8,04 & 8,09 & 7,91 \\
P6 & 7,84 & 7,74 & 7,75 & 7,84 & 8,09 \\
\hline
\end{tabular}

De acordo com Basso et al (2010) deve se ter atenção especial, quando a água utilizada na irrigação tem $\mathrm{pH}$ neutro, ou seja, quando as concentrações de $\mathrm{Ca}+\mathrm{Mg}$ e de bicarbonatos são maiores que 50 e $150 \mathrm{mg} / \mathrm{dm}^{3}$ (ppm), respectivamente. Resaltam que o ácido fosfórico não pode ser injetado via água de irrigação que contenha mais que $50 \mathrm{mg} \cdot \mathrm{dm}^{-3}$ (ppm) de cálcio e nitrato de cálcio, bem como em água que contenha mais de 5,0 meq. $\mathrm{L}^{-1}$ de $\mathrm{HCO} 3$, pois poderá formar precipitados de fosfato de cálcio.

Analisando os resultados da água observam-se as variações nos seguintes parâmetros de cálcio (mmolc L L ${ }^{-1}$ ) 0,07-0,40; magnésio $\left(\mathrm{mmolc} \mathrm{L}^{-1}\right)$ - 0,06-0,36; sódio $\left(\mathrm{mmolc} \mathrm{L}^{-1}\right)$ 0,47 - 3,64; potássio (mmolc $\mathrm{L}^{-1}$ ) - 0,03 - 0,27; cloreto $\left(\mathrm{mmolcL}^{-1}\right)$ - 0,14 - 1,30; sulfato (mmolc $\left.\mathrm{L}^{-1}\right)$ - 0,02 - 0,22; carbonatos (mmolc $\left.\mathrm{L}^{-1}\right)$ - 0,61 1,77 e bicarbonatos (mmolc $\mathrm{L}^{-1}$ ) - 0,03 - 0,29.

Em relação a esses parâmetros, as águas dos açudes estudados entre o período de setembro de 2009 e abril de 2010 encontram-se, com os valores normais para irrigação, exceto para os parâmetros cálcio, magnésio, cloreto e carbonatos (onde os valores normais são respectivamente de $0-20 ; 0-5 ; 0-30 ; 0-0,1$, de acordo com os valores normais em água de irrigação de Ayres \& Westcoot (1999).

Embora essas características usadas isoladamente não sejam suficientes para definir a qualidade da água para a irrigação, é importante ressaltar que águas com íons em excesso, podem trazer sérios prejuízos, principalmente quando se usa agricultura com irrigação localizada, já que o excesso de carbonatos provoca encrostamentos nos equipamentos de irrigação, e consequentemente causa entupimentos nas tubulações.

As variações observadas nos resultados, entre o inicio e o fim do monitoramento, provavelmente ocorreram pela acumulação de água no período chuvoso, alternado com redução por perdas pelo consumo e aumento de evaporação na estação seca, promovendo assim uma variação quantitativa e qualitativa no teor de sais das águas.

Os resultados obtidos indicam a necessidade de um manejo adequado da água desses açudes, de maneira que não venha futuramente comprometer as atividades agrícolas da região. As implicações negativas da salinidade 
estão relacionadas ao desenvolvimento e rendimento das plantas, podendo inclusive, comprometer a própria estrutura do solo, pois a absorção de sódio pelo solo, proveniente de águas com elevados teores deste íon, provoca a dispersão das frações de argila e, portanto, diminui a permeabilidade do solo.

\section{CONCLUSÕES}

No uso destas águas é de fundamental importância o uso de tratamentos, principalmente através da aplicação de gesso e lixiviação de sais, para um bom desenvolvimento e conservação das áreas agricultáveis. Além do mais, este estudo mostra a necessidade de monitoramento desta bacia para tomadas de decisões e elaboração de ações e políticas publicas agrícolas para o melhor desempenho da agricultura local.

As condições climáticas predominantes na região favorecem para que as águas dos açudes monitorados apresentassem um maior risco de salinidade e sodicidade ao longo do ano no período de temperaturas mais elevadas.

\section{AGRADECIMENTOS}

Os autores agradecem ao Conselho Nacional de Desenvolvimento Cientifico e Tecnológico ( $\mathrm{CNPq}$ ) e ao Instituo Federal de Educação, Ciência e Tecnologia do Ceará (IFCE), Campus Sobral, pelo apoio financeiro e estrutural para esta pesquisa.

\section{REFERÊNCIAS}

AYERS, R. S.; WESTCOT, D. W. A qualidade da água na agricultura, Campina Grande: UFPB, 1999. 153p. (Estudos FAO: Irrigação e Drenagem, 29 Revisado 1).

BERNARDO, S.; SOARES, A. A.; MANTOVANI, E. C. Manual de irrigação. 8 ed. - Viçosa - MG: Ed. UFV, 625 p. 2006.

BASSOI, L. H.; BRAGA, M.B.; CALGARO; M.; SIMÕES; W. L.; PINTO, J. M.; Irrigação e fertirrigação. In: Leão, P.C. de S.; Soares, J.M. (Org.) Cultivo da videira Petrolina: Embrapa Semiárido, 2010 (Sistema de Produção, 1 - 2a. edição)

CONAMA (Conselho Nacional Do Meio Ambiente). Resolução CONAMA n ${ }^{\circ} 357$ de 17 de março de 2005. Brasília: D.O.U., 2005. Disponível em: http://www.mma.gov.br/port/ conama/. Acesso em 04 de outubro de 2010.

CORDEIRO, G. G. Qualidade de água para fins de irrigação (Conceitos básicos e práticas). Petrolina, PE: Embrapa Semiárido. Documentos; 167, 32p. 2011

EATON, F.M. Significance of carbonates in irrigation waters. Soil Science, Baltimore, v.60, p.123-133, 1949.

ELOI, W. M. ; BARRETO, F. M. de S. . Qualidade microbiológica da água. In: Nildo da Silva Dias; Márcia Regina Farias da Silva; Hans Raj Gheyi. (Org.). Recursos Hídricos: usos e manejos. 1ed. São Paulo: Editora Livraria da Física, 2011, v., p. 129-148.

GHEYI, H. R.; DIAS, N. da S.; LACERDA, C. F. de. Manejo da salinidade na agricultura: Estudos básicos e aplicados. Fortaleza, INCT Sal, 472p, 2010.

IPECE - INSTITUTO DE PLANEJAMENTO DO CEARÁ. Anuário estatístico do Ceará 2002. (cd room). Governo do Ceará: Fortaleza, 2002. 
MOURA, R. S.; HERNANDEZ, F. B. T.; LEITE, M. A.; FRANCO, R. A. M.; FEITOSA, D. G.; MACHADO, L. F. Qualidade da água para uso em irrigação na microbacia do córrego do Cinturão Verde, município de Ilha Solteira. Revista Brasileira de Agricultura Irrigada v.5, n. 1, p.68-74, 2011

REICHARDT, K. A água em sistemas agrícolas. Editora Manole LTDA, 1990, primeira edição, 188p.

RICHARDS, L. A. (ed.) Diagnosis and improvement of saline and alkali soils. USDA Agricultural handbook 60. Washington: U.S: Department of Agriculture, 1954. 160 p.
SANTOS, J. R. G. A salinidade na agricultura irrigada: teoria e prática. Campina Grande, $\mathrm{PB}$ : UFPB, 2000. $171 \mathrm{p}$.

WILCOX, L.V., DURUM, W.H. Quality of irrigation water. In. HAGAN, R.M., HAISE, R.H., EDMINISTER, T.W. (ed.) Irrigation of agricultural lands. Madison: American Society of Agronomy, 1967 Chapter 9. p. 104 - 122. (Agronomy, 11).

YARON, B. Water suitability for irrigation. In: Yaron, E.; Danfors, E.; Vaadid, Y. (eds.). Arid zone irrigation. Berlin: Springler-Verlag, 1973. p.71-85. 